VIII Congresso Brasileiro de Informática na Educação (CBIE 2019)

Anais do XXV Workshop de Informática na Escola (WIE 2019)

\title{
Análise de Jogos em sites Educativos
}

\author{
Jefferson da $\operatorname{Rosa}^{1}$, Dulce Márcia Cruz ${ }^{1}$ \\ ${ }^{1}$ Programa de Pós-Graduação em Educação - Universidade Federal de Santa \\ Catarina (UFSC) - Florianópolis - SC - Brasil \\ jeffdarosa@hotmail.com, dulce.marciaegmail.com
}

\begin{abstract}
Nowadays many teachers would like to use digital games to contemplate subjects worked in the classroom. However, they find it difficult both because they do not know the sites that offer educational games and because they do not understand the main features of the games offered. This research aims to contribute to a better understanding of the content of websites classifying their games according to their mechanics, being a determining element for interaction with the game, because through it the player performs his actions. After a descriptive analysis of the websites and educational games available, four common mechanics were found that imply a specific mode of player interaction with the game.
\end{abstract}

Resumo: Atualmente muitos professores gostariam de utilizar jogos digitais para contemplar temas trabalhados em sala de aula. Porém, encontram dificuldades tanto por não conhecerem os sites que oferecem jogos educativos quanto por não entenderem as principais características dos games oferecidos. Esta pesquisa tem como objetivo contribuir para uma melhor compreensão do conteúdo dos sites classificando os seus jogos de acordo com a sua mecânica, sendo ela um elemento determinante para a interação com o jogo, pois através dela o jogador realiza suas ações. Após uma análise descritiva dos sites e dos jogos educativos disponíveis foram encontradas quatro mecânicas mais comuns que implicam num modo específico de interação do jogador com o jogo.

\section{Introdução}

Muito se discute a ideia de que a aplicação de jogos digitais no contexto escolar poderia ser uma alternativa bem sucedida no processo de ensino e aprendizagem, pois muitas crianças têm acesso aos games em seus momentos de lazer através de consoles, computadores, tablets e smartphones, empregando grande parte do seu tempo jogando. $\mathrm{O}$ argumento principal para sua função educativa seria que o uso desses artefatos poderia atrair a atenção dos alunos em uma determinada aula, visto que boa parte deles já está acostumada com os jogos digitais em seu cotidiano.

Os jogos digitais utilizados nas escolas são em geral considerados "educativos", porque geralmente trazem conteúdos escolares, ou seja, são jogos de Matemática, Português, Ciências, Geografia, entre outros, que os professores escolhem porque contemplam de alguma forma os temas trabalhados em sala de aula. De acordo com Schell (2011) ,os jogos são compostos de narrativas, estética, tecnologia e mecânica. Ter conhecimento sobre esses elementos seria útil para ampliar as possibilidades de prática de jogos digitais no contexto escolar. O problema é que professores não sabem onde encontrar os jogos ou, se os encontram, não são capazes de escolher e identificar 
VIII Congresso Brasileiro de Informática na Educação (CBIE 2019)

Anais do XXV Workshop de Informática na Escola (WIE 2019)

os elementos que poderiam justificar seu uso na escola. Além disso, por não conhecer o que são jogos digitais, muitos professores acabam, por segurança, tentando escolher aqueles apresentados no mercado como educativos, geralmente disponíveis em sites específicos, que trazem uma grande variedade de opções, de forma gratuita e aberta, mas que, pela enorme quantidade de oferta, em vez de facilitar, podem dificultar a escolha do que usar.

Uma rápida navegada na internet mostra que os sites que oferecem jogos educativos casuais em diferentes gêneros (puzzle, casuais, cognitivos, entre outros) não trazem informação útil para os professores, tais como, por exemplo, que conteúdos são trabalhados, que habilidades ou competências podem desenvolver, para qual nível de ensino ou faixa etária seriam mais adequados ou mesmo para quais objetivos pedagógicos poderiam ser utilizados. Sem essas informações, os professores, que em muitos casos não costumam ser jogadores, ficam inseguros, optando pela não utilização do recurso, justamente pela falta de domínio e conhecimento. Ou, quando escolhem, não conseguem ter certeza do quanto o jogo efetivamente contribuiu para a aprendizagem de seus estudantes e se conseguiram corresponder à sua intencionalidade.

Pensando em contribuir para essa situação problemática, foi realizada uma pesquisa para compreender um pouco melhor quais são os principais sites que oferecem jogos educativos digitais, como se estruturam, o que oferecem de informação, como são por dentro os jogos disponíveis e classificá-los de acordo com suas mecânicas.

Para isso, este artigo está dividido da seguinte forma: a seção 2 apresenta uma revisão da literatura abordando análises de jogos educativos. A seção 3 exibe uma fundamentação teórica sobre o conceito de jogos, jogos digitais, jogos educativos e mecânicas de jogos. A seção 4 descreve a metodologia utilizada para analisar jogos em sites educativos. A seção 5 aponta os resultados encontrados na pesquisa. E na seção 6 encontram-se as considerações finais do trabalho exposto.

\section{Trabalhos Relacionados}

Como pretendemos fazer análise de jogos educativos, realizamos uma busca para encontrar trabalhos afins que nos ajudassem a responder ao objetivo da pesquisa. Vamos citar os três textos encontrados com maior proximidade com nossa temática. $\mathrm{O}$ primeiro é o trabalho de Calisto et al. (2010), no qual os autores compararam jogos educativos, examinando os seguintes critérios: a finalidade do jogo, o público alvo, a tecnologia e avaliação. A partir dessa análise, apresentam o desenvolvimento de um jogo digital para a educação ambiental, com o objetivo de aumentar o interesse e a motivação dos alunos.

No trabalho de Medeiros e Schimiguel (2012) é mostrada uma análise do uso de jogos eletrônicos na educação como auxílio no processo de aprendizagem. Também é apresentada uma revisão de literatura sobre os benefícios dos jogos educacionais e considerações na aplicação de jogos eletrônicos nas escolas. No final, o autor sugere uma abordagem para avaliação de jogos eletrônicos educacionais.

Já o trabalho de Santos et al. (2015) avalia dois jogos educativos, em aspectos computacionais de interface e aspectos pedagógicos, de forma quantitativa e qualitativa, baseando-se em três diferentes abordagens para a avaliação de jogos (LORI, GameFlow e modelo de Kirkpatrick). De acordo com os autores, os jogos tiveram resultados positivos diante do cenário em que foram avaliados e o processo de avaliação adotado 
VIII Congresso Brasileiro de Informática na Educação (CBIE 2019)

Anais do XXV Workshop de Informática na Escola (WIE 2019)

pode ser replicado ou adaptado para outros jogos educativos, mesmo que estes sejam de domínios diferentes.

Apesar dos textos citados serem exemplo de outras pesquisas semelhantes de avaliação de jogos, não foram encontradas referências de análise de sites comerciais e dos jogos educativos disponíveis que pudessem ser utilizadas como parâmetro para responder ao nosso objetivo principal.

\section{Elementos que constituem os jogos}

Para realizar uma análise dos jogos educativos é preciso inicialmente definir o que são eles. Huizinga, em sua obra Homo Ludens, define o jogo como

uma atividade ou ocupação voluntária, exercida dentro de certos e determinados limites de tempo e de espaço, segundo regras livremente consentidas, mas absolutamente obrigatórias, dotado de um fim em si mesmo, acompanhado de um sentimento de tensão e de alegria e de uma consciência de ser diferente da "vida quotidiana" (2000, p.24).

Callois (1990), por sua vez, aponta o jogo como uma atividade livre, delimitada, incerta, improdutiva, regulamentada e fictícia. Salen \& Zimmerman (2012) definem o jogo como um sistema onde os jogadores participam de um conflito artificial, com regras, que provoca um resultado quantificável.

Os jogos que são produzidos especialmente para aparelhos eletrônicos, como computadores, celulares, videogames, entre outros, são conhecidos como jogos digitais, também podem ser chamados de jogos eletrônicos, jogos virtuais, games, entre outros. Todas as características atribuídas ao conceito de jogos, apresentadas anteriormente, também servem para descrever os principais aspectos dos jogos digitais. Porém, uma de suas principais especificidadesé a utilização de aparelhos digitais para a sua execução. Nesse sentido, Salen \& Zimmerman ressaltam que:

Os jogos digitais são sistemas, assim como todos os outros jogos [...]. O meio físico do computador é um elemento que compõe o sistema do jogo, mas não representa todo o jogo. O hardware e o software do computador são apenas os materiais dos quais o jogo é composto (2012, p. 102).

Schuytema (2008) define o jogo digital como uma atividade lúdica constituída de ações, decisões, regras e pelo universo do game, que resultam em uma condição final. As regras e o universo do game são demonstrados por meios eletrônicos e controlados por um programa digital.

Já os jogos digitais educativos constituem objetivos voltados para o aprendizado de determinados conteúdos pedagógicos. Segundo Ramos e Cruz (2018) são aqueles que têm finalidades educativas e podem ser definidos como "uma estratégia pedagógica que alia conhecimentos teóricos à prática da jogabilidade, oferecendo curiosidade e diversão ao possibilitar o engajamento dos envolvidos (2018, p. 61)". Para Novak:

Jogos educativos são aqueles criados para ensinar enquanto distraem. [...] desenvolvidos especificamente para fins pedagógicos, sempre com as crianças como público-alvo. [...] Esses games baseiam-se na aquisição de conhecimentos ao jogar, sendo que certos tópicos (como geografia, matemática e leitura) são ensinados ou acessados dentro do próprio game. $\mathrm{Na}$ 
VIII Congresso Brasileiro de Informática na Educação (CBIE 2019)

Anais do XXV Workshop de Informática na Escola (WIE 2019)

maioria dos games educativos, as matérias são ensinadas abertamente (2010, p. 75-76).

Os jogos proporcionam um ambiente seguro para que os jogadores aprendam e desenvolvam novas habilidades (RAMOS; CRUZ, 2018). De acordo com Schell (2011), o jogo digital é produzido com a intenção de promover experiências aos jogadores. $\mathrm{O}$ autor aponta que os jogos digitais possuem quatro elementos estruturantes que são fundamentais na sua constituição: a mecânica, a estética, a tecnologia e a narrativa. Mais do que a estética, a narrativa e a tecnologia, a mecânica poderia ser um dos elementos definidores do que seria um jogo educativo, pois é ela que determina os procedimentose o comportamento do jogador, o que ele precisa fazer para se relacionar com o game e quais são os seus objetivos. Ou, como Schell (2011), que aponta que a mecânica é encarregada por distinguir o jogo de outra mídia. Descreve as possibilidades de como os jogadores podem ou não conseguir alcançar os objetivos propostos pelo jogo. Para Chandler (2012), a mecânica do jogo abrange várias ações que o jogador executa ou vivencia no jogo. Alguns dos sistemas que se encaixam nessa categoria são: os desafios; as recompensas; a curva de aprendizado; o esquema de controle; as ações do jogador; e os elementos multijogador.

Pelo exposto, o elemento "mecânica" parece ser o mais promissor dentre os componentes do jogo para centrar o foco para uma análise da sua intencionalidade educativa. Isso porque a mecânica é responsável em estabelecer a interação entre o jogo e o jogador. De acordo com Salen \& Zimmerman (2012), jogar implica interatividade e, fazer escolhas, significa interagir. Partindo desse pressuposto, foi realizada uma primeira pesquisa exploratória, não sistemática e livre, em sites populares de jogos comerciais que apresentam de alguma maneira a categoria "educativos", para levantar se haveria mecânicas mais comuns nos games disponíveis. Como resultados indicativos foram encontrados quatro modos de interação principais: clicar com o mouse na resposta; digitar a resposta; arrastar a resposta; direcionar o personagem até a resposta. Constatou-se que os jogos considerados educativos nesses sites possuem ao menos uma das mecânicas citadas e que essas são as principais ações realizadas pelo jogador, para responder determinada pergunta durante o jogo. Para sistematizar esses achados foi realizada uma pesquisa cuja metodologia e resultados serão descritos a seguir.

\section{Metodologia}

Esta pesquisa caracteriza-se como exploratória, orientando-se pela abordagem qualitativa, visando conhecer melhor os jogos educativos presentes nos sites comerciais. Ela foi realizada entre os dias 02/01/2019 e 07/06/2019. O primeiro passo foi escolher quais sites educativos seriam analisados. $\mathrm{O}$ critério utilizado para definir os sites com jogos educativos que seriam investigados seguiu a lógica que os professores provavelmente utilizam para encontrar jogos para usar em suas atividades pedagógicas, ou seja, foi feita uma busca no Google com as seguintes palavras chave: "jogos educativos online", "jogos pedagógicos online" e "jogos escolares online". Como critério de exclusão, não consideramos os sites que não ofereciam jogos educativos.

O quadro 1 apresenta o nome dos sites encontrados após a busca, separados em colunas contendo as palavras-chave que os encontraram:

Quadro 1: Sites encontrados após as buscas

"jogos educativos online" $\quad$ "jogos pedagógicos online" "jogos escolares online" 
VIII Congresso Brasileiro de Informática na Educação (CBIE 2019)

Anais do XXV Workshop de Informática na Escola (WIE 2019)

\begin{tabular}{|l|l|l|}
\hline $1^{\circ}$ - Escola games & $1^{\circ}$ - Escola games & $1^{\circ}$ - Escola games \\
$2^{\circ}$ - Jogos grátis para Crianças & $2^{\circ}$ - SmartKids & $2^{\circ}$ - Jogos da escola \\
$3^{\circ}$ - SmartKids & $3^{\circ}$ - Jogos Educativos Online & $3^{\circ}$ - SmartKids \\
$4^{\circ}$ - Jogos Educativos Online & $4^{\circ}$ - Jogos grátis para Crianças & $4^{\circ}$ - Jogos Online grátis \\
$5^{\circ}$ - Hvirtua & $5^{\circ}$ Jogos 360 & $5^{\circ}$ - Hvirtua \\
$6^{\circ}-$ Jogos 360 & $6^{\circ}$ - Hvirtua & $6^{\circ}$ - Nova Escola \\
$7^{\circ}$ - Click Jogos & $7^{\circ}$ - Click Jogos & $7^{\circ}$ - Jogos 360 \\
$8^{\circ}$ - Poki & $8^{\circ}$ - Games Educativos & $8^{\circ}$ - Ludo Educativo \\
$9^{\circ}$ - Games Educativos & $9^{\circ}$ - Educa Jogos & $9^{\circ}$ JogosEducando.com \\
$10^{\circ}-$ Atividades Educativas & $10^{\circ}$ - Só Pedagogia & $10^{\circ}$ - Iguinho \\
\hline
\end{tabular}

Foram escolhidos para análise aqueles sites que apareceram nas três palavraschave: Escola Games, Smartkids, Hvirtua e Jogos 360. O procedimento seguido foi visitar esses sites, acessar e descrever todos os hiperlinks apresentados, quantificar os jogos e todos os materiais disponíveis, jogar os jogos da disciplina escolar que apresentava o maior número de jogos e os classificar de acordo com as suas mecânicas. Nos próximos itens será descrito cada um deles, mostrando como se estruturam, de que modo os jogos educativos aparecem para o jogador, que tipo de classificação interna possuem e como conduzem o internauta para encontrar o que procura para jogar.

\subsection{Escola games}

Ao abrir o site Escola Games foi possível observar subdivisões no topo da página: Fácil, Médio, Difícil, Jogos, Livros e Aplicativos.

Na opção "Fácil" foram encontrados 32 jogos, na opção "Médio" 53 jogos e em "Difícil" 17 jogos. Nelas os jogos foram classificados, de acordo com os autores, por nível de dificuldade. Nas três subdivisões supracitadas existe a opção "filtrar por matéria", que divide os jogos nas seguintes disciplinas: Artes, Ciências, Educação Física, Espanhol, Geografia, História, Inglês, Italiano, Língua Portuguesa e Matemática.

Na opção "Jogos" foram encontrados todos os jogos do site (93 jogos) e também a opção de filtrar por matéria disponível:
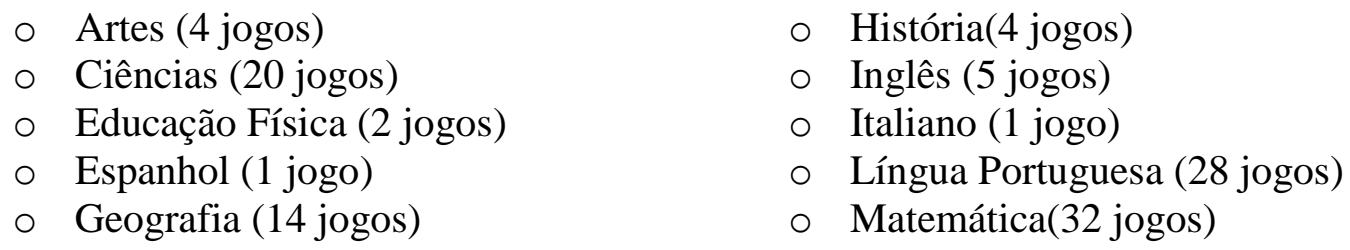

Na opção "Aplicativos" são mostrados 25 opções de aplicativos para serem baixados. Trata-se de jogos educativos que podem ser jogados em smartphones.

Após este levantamento, foram escolhidos para análise os jogos da área de conhecimento que apresentou o maior número de jogos. Este critério também será utilizado nos próximos sites apresentados. Neste, a Matemática foi a área de conhecimento que somou a maior quantidade de jogos (32) e todos eles foram jogados pelo menos uma vez.

O quadro2 apresenta os jogos de Matemática classificados de acordo com as mecânicas encontradas:

\section{Quadro 2: Jogos de Matemática do site Escola Games}

\begin{tabular}{l|l} 
Mecânica & Título dos Jogos \\
\hline
\end{tabular}


VIII Congresso Brasileiro de Informática na Educação (CBIE 2019)

Anais do XXV Workshop de Informática na Escola (WIE 2019)

\begin{tabular}{|c|l|}
\hline $\begin{array}{c}\text { Clicar na } \\
\text { resposta }\end{array}$ & $\begin{array}{l}\text { Circo Mágico; Algarismos Romanos; Coelho da Páscoa; Casa da Carne; Batalha dos } \\
\text { Números; Antecessor e Sucessor; Coral Didático; Dividindo a pizza*; Operação } \\
\text { Páscoa; Aprenda a Contar; Calendário Mágico; Maior e Menor da Selva; Par ou } \\
\text { Ímpar; Eu sei Contar; Zoo Louco; Tabuada do Dino*; Completando os Números*; } \\
\text { Formas Geométricas*; Jogo da Fauna; Jogo da Independência. }\end{array}$ \\
\hline $\begin{array}{c}\text { Digitar a } \\
\text { resposta }\end{array}$ & $\begin{array}{l}\text { Robô Lógico*; Dividindo a pizza*; Tabuada do Dino*; Blocos espaciais; } \\
\text { Completando os Números*. }\end{array}$ \\
\hline $\begin{array}{c}\text { Arrastar a } \\
\text { resposta }\end{array}$ & $\begin{array}{l}\text { Zebuzim; Quebra-cabeça; Aprendendo as Horas; Formas e Desenhos; Quebra-cabeça } \\
\text { (Turma do Paulinho); Trânsito Legal. }\end{array}$ \\
\hline $\begin{array}{c}\text { Direcionar o } \\
\text { personagem até } \\
\text { a resposta }\end{array}$ & $\begin{array}{l}\text { Mestre das Tabuadas; Vôo Educativo; Labirinto Polar; Robô Lógico*; Pega Bolhas; } \\
\text { Formas Geométricas*. }\end{array}$ \\
\hline
\end{tabular}

*Jogos que utilizam duas ações para responder determina pergunta.

Após jogar os 32 jogos de Matemática foi possível constatar que todos os jogos se encaixam, no mínimo, em uma mecânica citada. Têm jogos que utilizam mais de uma mecânica no decorrer de seu jogo.

\subsection{SmartKids}

Na tela inicial do site SmartKids é possível observar seis subdivisões: Jogos, Para Colorir, Atividades, Trabalhos Escolares, Datas e Vídeos.

Na subdivisão "Jogos" foram encontrados alguns jogos em destaque, porém, no canto superior direito é mostrado um botão escrito "categorias", responsável por dividir todos os jogos (total de 712), e mostrar a quantidade de cada agrupamento:

$\begin{array}{ll}\circ & \text { Alfabeto: } 5 \\ \circ & \text { Animais: } 36 \\ \circ & \text { Come come: } 4 \\ \circ & \text { Ditado: } 8 \\ \circ & \text { Esportes: } 24 \\ \circ & \text { Habilidade: } 65 \\ \circ & \text { Higiene Bucal: } 12 \\ \circ & \text { Inglês: } 4 \\ \circ & \text { Jogo da Forca: } 52 \\ \bigcirc & \text { Jogo dos Erros: } 6 \\ \bigcirc & \text { Jogos da Memória: } 83 \\ \bigcirc & \text { Jogos de Cenário: } 33 \\ \bigcirc & \text { Jogos de Cozinhar: } 39\end{array}$

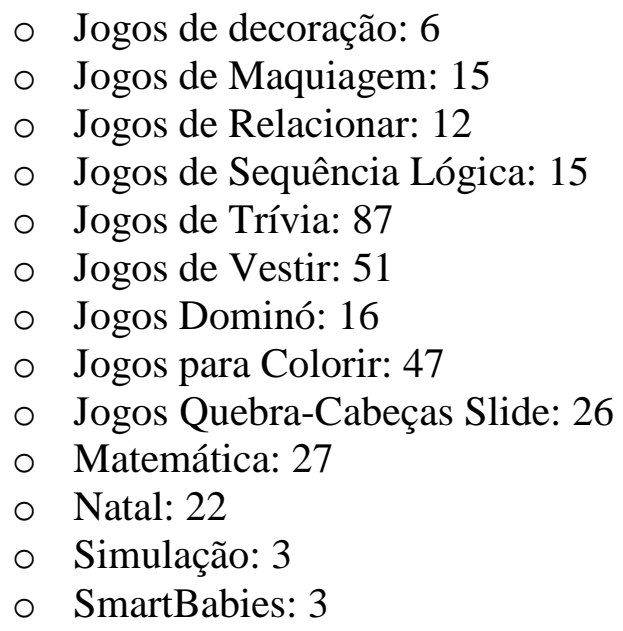

Neste site os jogos não são divididos por área de conhecimento, e sim por diversas categorias, como: esportes, forca, quebra-cabeça, natal, entre outros. Encontram-se duas categorias representadas por área de conhecimento, Inglês e Matemática, contendo 4 e 27 jogos, respectivamente cada uma. Então, foram escolhidos os jogos de Matemática para serem jogados e analisados, pois é a disciplina escolar que contém o maior número de jogos neste site, conforme o quadro3:

\section{Quadro 3: Jogos de Matemática do site SmartKids}

\begin{tabular}{|c|l|}
\hline Mecânica & \multicolumn{1}{c|}{ Título dos Jogos } \\
\hline Clicar na & Calcule e Pinte Higiene Bucal;Cantina dona Maria;Formas Geométricas:Jogo da \\
resposta & Adição;Jogo da Multiplicação;Jogo da Subtração;Operações na Selva;Sequência;Jogo \\
& de Xadrez;Formas Geométricas Cálculo;Jogo Matemática Operações;Jogo Operações \\
& $\begin{array}{l}\text { Borboleta*; Multiplicação e Divisão dos } \\
\text { Monstros;OperaçõesEspaço;MatemáticaDivertida;OperaçõesBaseball;JogoSudoku;Ta } \\
\end{array}$ \\
& buada do 6;Tabuada do 7;Tabuada do 8;Tabuada do 9. \\
\hline Digitar a & Jogo Operações Borboleta*; \\
\hline
\end{tabular}


VIII Congresso Brasileiro de Informática na Educação (CBIE 2019)

Anais do XXV Workshop de Informática na Escola (WIE 2019)

\begin{tabular}{|c|l|}
\hline resposta & \\
\hline $\begin{array}{c}\text { Arrastar a } \\
\text { resposta }\end{array}$ & $\begin{array}{l}\text { Adição Descubra;Mova Palito;Ordenar Números;Vamos Contar;Pincel Mágico; } \\
\text { Medidas e Grandezas. }\end{array}$ \\
\hline $\begin{array}{c}\text { Direcionar o } \\
\text { personagem até } \\
\text { a resposta }\end{array}$ & \\
\hline \\
*Jogos que utilizam duas ações para responder determina pergunta.
\end{tabular}

\subsection{HVirtua}

No topo do site HVirtua foram encontradas as seguintes subdivisões: Maternal, Jardim, Pré-Escolar, Feitos por Hvirtua e Contato.

- Maternal: Esta categoria apresenta 11 jogos, sendo em sua maioria jogos de colorir e de encaixar formas.

- Jardim: Nesta seção existem 16 jogos. São mostrados jogos de colorir, encaixar formas, quebra-cabeça e jogos que envolvem as vogais.

- Pré-Escolar: Este segmento contém 29 jogos. Aqui, a maioria dos jogos é composta por temas que envolvem alfabetização e jogos de matemática.

- Feitos por Hvirtua: Este espaço é constituído por 32 jogos produzidos pelos autores do site. Muitos destes jogos estavam presentes nas seções anteriores.

- Contato: Aqui há uma área destinada à comunicação com os produtores. São disponibilizados e-mail, página do Facebook e campo de mensagem. Existe também um espaço aberto para comentários que os usuários podem utilizar.

Este site também não possui uma divisão dos jogos por área de conhecimento. Para realizar uma divisão, jogamos todos os jogos deste site e categorizamos nas disciplinas escolares de acordo com as suas características. Logo, foi possível constatar que existem 12 jogos de Português, cinco de Matemática, um de Inglês e um de Geografia. Sendo assim, jogamos todos os jogos de Português disponíveis no site e analisamos de acordo com as suas mecânicas, conforme o quadro 4:

Quadro 4: Jogos de Português do site HVirtua

\begin{tabular}{|c|l|}
\hline Mecânica & \multicolumn{1}{|c|}{ Título dos Jogos } \\
\hline $\begin{array}{c}\text { Clicar na } \\
\text { resposta }\end{array}$ & $\begin{array}{l}\text { Atividades diversas; Parquinho das Vogais; Memória Alfabeto; Brincando e } \\
\text { Associando; Vogais e Figuras 2; Vogais e Figuras; Jogo da Memória; ABCDario. }\end{array}$ \\
\hline $\begin{array}{c}\text { Digitar a } \\
\text { resposta }\end{array}$ & \\
\hline $\begin{array}{c}\text { Arrastar a } \\
\text { resposta }\end{array}$ & Sílabas e Figuras; Jogo do Alfabeto; Encaixa Vogais. \\
\hline $\begin{array}{c}\text { Direcionar o } \\
\text { personagem até } \\
\text { a resposta }\end{array}$ & PulABC \\
\hline
\end{tabular}

\subsection{Jogos 360}

Este site, de acordo com os seus produtores, possui mais de 10.000 jogos, e todos os dias são inseridos novos jogos. No topo da tela inicial há quatro subdivisões: Top Jogos, Novos Jogos, Categorias e Super Listas. Ao lado encontra-se uma ferramenta de busca, para que o usuário possa pesquisar algum conteúdo específico no site.

- Top Jogos: Nesta seção foram encontrados 660 telas, quase todas possuem 30 jogos cada, pois as telas 659 e 660 contêm 27 e 29 jogos respectivamente. Totalizando 19.796 jogos nesta subdivisão. 
VIII Congresso Brasileiro de Informática na Educação (CBIE 2019)

Anais do XXV Workshop de Informática na Escola (WIE 2019)

- Novos Jogos: Aqui são apresentadas 663 telas. A última tela contém 16 jogos, todas as outras dispõem de 30 jogos. Somando tudo totalizou 19.876 jogos nesta categoria.

- Categorias: Neste espaço os jogos são divididos por categorias e sub-categorias, alguns são conhecidos pelo nome, boa parte em inglês. Outros são nomeados de variadas maneiras, que vão desde gêneros tradicionais como aventura e corrida a outras classificações inusitadas, tais como, por exemplo, "meninas", "vestir e maquiar", claramente sexistas.

- Aventura (14008 jogos)

○ Corrida (2719 jogos)

- Clássicos (922 jogos)

- Vestir e Maquiar (7926 jogos

○ Habilidade (3354 jogos)

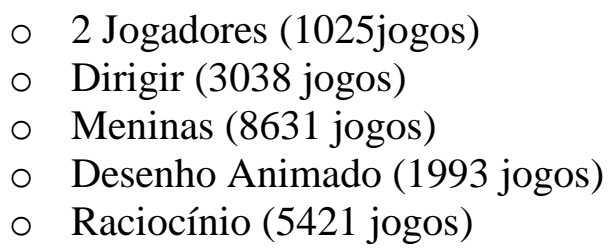

- Super Listas: São 85 listas selecionadas pela equipe produtora do site, com jogos de personagens populares (Mário, Minecraft, Sonic, Pokémon, Barbie, entre outros), jogos de RPG, jogos de luta, jogos de futebol, entre outros.

Abaixo dessas quatro seções, o site apresenta outros agrupamentos. Em alguns deles também foram encontrados uma subdivisão por "categorias": 2 Jogadores (813 jogos), Vestir (2.472 jogos), Carro (1.125 jogos), Cozinhar (438 jogos), Futebol (398 jogos), Luta (1.248 jogos), Meninas (3.711 jogos), Tiro (2.176 jogos), Moto (560 jogos) e Corrida (1.134 jogos).

Neste site, entre as diversas categorias e sub-categorias apresentadas, a única disciplina escolar que aparece como categoria é a Matemática. Então, utilizamos os seus jogos (48) para jogar e classificar de acordo com as suas mecânicas, conforme o quadro5:

\section{Quadro 5: Jogos de Matemática do site Jogos 360}

\begin{tabular}{|c|l|}
\hline Mecânica & \multicolumn{1}{c|}{ Título dos Jogos } \\
\hline resposta & $\begin{array}{l}\text { GroceryCashier; One Plus Two; FrozenSchool; Cowboy Zombie; The Operator; } \\
\text { SupermarketCount; 1+2=3; Math Game for Kids; PeppaPigIceskating; Sevenventure; } \\
\text { PinteMilliEnsinandoosNúmeros; Math Game; Ben 10: SkaterMath; Descendants - } \\
\text { Puzzle, MessyandMath; Soy Luna Math Quis; Math Mountain; Numbers; Double } \\
\text { Digits; Pathways; Pathways; MatHematics; BrainSpa 2; } \\
\text { PregnantAngelaSchoolTeacher; InazumaHaveProblems; JakePirateSchool; } \\
\text { FindingandReleasingDory; The Idiot Test 4; Plupon; Towerblaster; AddLikeMad; } \\
\text { Popoint; Plus!Plus!; ArmorPicross; Multiplication; The 43-seconds Game; The } \\
\text { Equator; DefeatYourFriend*; Dora andMermaidBirthday; BrainMachine; } \\
\text { NumbersCompetition; Pregnant Rapunzel SchoolTeacher; PinteCaillou e Clementine; } \\
\text { Koala Kid. }\end{array}$ \\
\hline $\begin{array}{c}\text { Digitar a } \\
\text { resposta }\end{array}$ & MathHurdles. \\
\hline $\begin{array}{c}\text { Arrastar a } \\
\text { resposta }\end{array}$ & Solve Math. \\
\hline $\begin{array}{c}\text { personagem até } \\
\text { a resposta }\end{array}$ & 2048; MaximumRotation; DefeatYourFriend*; Pochaccos Bank. \\
\hline
\end{tabular}

*Jogos que utilizam duas ações para responder determina pergunta.

\section{Resultados}

Após jogar todos os 126 jogos escolhidos dos quatro sites e classificá-los de acordo com as suas mecânicas, tivemos os seguintes resultados: 
VIII Congresso Brasileiro de Informática na Educação (CBIE 2019)

Anais do XXV Workshop de Informática na Escola (WIE 2019)

Tabela 1: Mecânicas encontradas nos jogos pesquisados

\begin{tabular}{|l|c|c|c|c|}
\hline & Clicar na resposta & Digitar a resposta & Arrastar a resposta & $\begin{array}{c}\text { Direcionar o } \\
\text { personagem até a } \\
\text { resposta }\end{array}$ \\
\hline Escola Games & 20 & 5 & 6 & 6 \\
\hline SmartKids & 20 & 1 & 6 & 0 \\
\hline HVirtua & 8 & 0 & 3 & 1 \\
\hline Jogos 360 & 43 & 1 & 1 & 4 \\
\hline Total: & $\mathbf{9 1}$ & $\mathbf{7}$ & $\mathbf{1 6}$ & $\mathbf{1 1}$ \\
\hline
\end{tabular}

Observando a tabela é possível perceber que a maioria dos jogos faz uso da mecânica de clicar na resposta, para que o usuário consiga interagir com o jogo. Será utilizado agora o site "Escola Games" para analisar os jogos que possuem essa mecânica (Vide Quadro 1). Foi escolhido esse site pelo fato de ele aparecer em primeiro nas buscas do Google, através das palavras chaves e, pela mesma lógica de tentar seguir o senso comum que é a de levar em conta a tendência dos internautas de irem ao primeiro resultado que aparece. Optou-se pela mecânica de "Clicar na Resposta", como um recorte possível, pois é a ação encontrada na maioria dos jogos aqui analisados.

No site Escola Games foram encontrados 20 jogos de Matemática que apresentam a mecânica de clicar na resposta. Essa mecânica retrata diferentes particularidades em alguns jogos. Em nove jogos, por exemplo, existe uma ação onde o jogador precisa clicar na resposta (ou montá-la) e, em seguida, clicar em "conferir". Se a resposta estiver correta, geralmente, aparece uma mensagem confirmando o acerto. Se estiver errada é mostrado para o jogador que a resposta está incorreta. Em outros sete jogos, o jogador precisa clicar diretamente na resposta, sem a necessidade de clicar em algum botão para confirmar a resposta. Nesse caso, também há mensagens expondo se a resposta está correta ou incorreta.

Apesar dos jogos possuírem o mesmo tipo de mecânica, a estética e a narrativa são os elementos que diferem um jogo de outro. Através desses atributos percebemos que a sequência de eventos, a aparência e os sons são também características essenciais que garantem a singularidade em cada game educativo. Foram também encontrados dois jogos onde o jogador precisar clicar em cima de objetos para contá-los, obedecendo à uma sequência numérica. E dois jogos de memória, no qual é preciso o usuário clicar sobre as cartas para encontrar os seus pares.

\section{Considerações Finais}

Este artigo trouxe como contribuição a análise detalhada de quatro sites populares que oferecem jogos educativos online. Entendemos que para o professor utilizar jogos no contexto escolar, ele precisa ter conhecimento sobre o objetivo pedagógico do site e sobre a equipe de produção que oferta esses materiais, pois é relevante saber se são elaborados por profissionais compromissados com a educação, com o intuito de atingir determinadas finalidades pedagógicas.

O presente trabalho pôde verificar que os quatro tipos de mecânicas ocorrem na maioria dos jogos digitais educativos, com conteúdos escolares. E em alguns deles essas mecânicas não aparecem de maneira isolada, ou seja, duas ou mais ações são realizadas pelo usuário enquanto ele interage com o jogo. Foi possível constatar que a grande maioria dos jogos educativos analisados (76\%) faz uso da mecânica de clicar com o mouse na resposta, isto é, para solucionar determinado problema exposto durante o jogo, o usuário precisa clicar na resposta, ou montá-la, para poder prosseguir no jogo. Geralmente, após essa tomada de decisão, aparece uma mensagem mostrando para o 
jogador se a resposta está correta ou não.

É importante ressaltar que essa pesquisa é exploratória, feita para apontar alguns indicadores para servir de apoio aos professores, mas ainda é preciso avançar mais. Da mesma maneira que foi realizada, seria interessante investigar como os professores pesquisam os jogos, como interpretam as informações disponíveis nos sites, quais parâmetros seguem para definir suas escolhas e os planejamentos de uso dos jogos escolhidos. Ainda seria válido verificar se essas mecânicas mais comuns são realmente recorrentes, se possuem um potencial educativo que justifique sua maior presença na oferta. Ou mesmo, se respondem às necessidades dos professores em seus planejamentos. Outra limitação da pesquisa é que não foi utilizada a palavra-chave "game" e seus derivados em inglês. Como esse levantamento exploratório faz parte de uma pesquisa de mestrado em andamento, iremos realizar novas pesquisas para tentar verificar se essa inclusão pode trazer diferença nos resultados.

Como conclusão,pode-se afirmar que é preciso apoiar os professores para utilizarem jogos digitais educativos em suas aulas, mas, para isso, precisam compreender melhor suas principais características, perpassando pelos diferentes tipos de mecânicas existentes e uma possível relação entre elas e a aprendizagem pretendida.

\section{Referências}

CAILlOIS, Roger. (1990) Os jogos e os Homens:A máscara e a vertigem. Lisboa: Edições Cotovia.

CALiSTO A.; BARBOSA D.; SILVA C. (2010) Uma Análise Comparativa entre Jogos Educativos Visando a Criação de um Jogo para Educação Ambiental. XXI Simpósio Brasileiro de Informática na Educação. João Pessoa: Sociedade Brasileira de Computação - SBC.

CHANDLER, Heather Maxwell. Manual de Produção de Jogos.Bookman. Porto Alegre, 2012.

HUIZINGA, J. (2010) Homo Ludens. São Paulo:Editora Perspectiva.

MEDEIROS, M., O.; SCHIMIGUEL, J. (2012) Uma Abordagem Para Avaliação De Jogos Educativos: Ênfase No Ensino Fundamental. XXIII Simpósio Brasileiro de Informática na Educação. Rio de Janeiro: Sociedade Brasileira de Computação SBC.

NOVAK, Jeannie.(2010)Desenvolvimento de Games. São Paulo: CengageLearning.

RAMOS, D. K.; CRUZ, D. M. (Org.) (2018). Jogos digitais em contextos educacionais. Curitiba: CRV.

SALEN, K. ZIMERMAN, E. (2012) Regras do Jogo. Fundamentos do Design de Jogos. São Paulo:Blucher.

SANTOS W. O.; NETO. S. R. S.; JUNIOR C. G. S.; BITTENCOURT I. I. (2015) Avaliação de Jogos Educativos: Uma Abordagem no Ensino de Matemática. XXVI Simpósio Brasileiro de Informática na Educação. Maceió: Sociedade Brasileira de Computação - SBC.

SCHELL, Jesse. (2011) The Art of Game Design: A Book of Lenses. Elsevier Morgan Kaufman Publishers.

SCHUYTEMA, Paul. (2008) Design de games: uma abordagem prática. Cengage Learning. 